\title{
Critical Behavior of Coupled q-state Potts Models under Weak Disorder
}

\author{
P. Simon用 \\ Laboratoire de Physique Théorique et Hautes Energies \ \\ Universités Pierre et Marie Curie Paris VI et Denis Diderot Paris VII \\ 2 pl. Jussieu, 75251 Paris cedex 05
}

\begin{abstract}
We investigate the effect of weak disorder on different coupled $q$-state Potts models with $q \leq 4$ using two loops renormalisation group. This study presents new examples of first order transitions driven by randomness. We found that weak disorder makes the models decouple. Therefore, it appears that no relations emerge, at a perturbation level, between the disordered $q_{1} \times q_{2}$-state Potts model and the two disordered $q_{1}$, $q_{2}$-state Potts models $\left(q_{1} \neq q_{2}\right)$, despite their central charges are similar according to recent numerical investigations. Nevertheless, when two $q$-state Potts models are considered $(q>2)$, the system remains always driven in a strong coupling regime, violating apparently the Imry-Wortis argument.
\end{abstract}

PACS NUMBERS: 05.70.Jk; 64.60.Fr; 75.10.Hk; 75.40.Cx

*simon@lpthe.jussieu.fr

† Unité associée au CNRS URA 280 
The effect of weak randomness on continuous phase transitions has been much studied either analytically [1, 2], or numerically [3]. For most of these cases, the Harris criterion [4] provides us a good method to see if disorder will change the universality class of the transition. However, it has been realized recently, that a weak bond randomness can also have strong effects on first order transitions $2 D$ systems and can induce a second order phase transition in a system that would undergo a first order one [5]. This result has been established more rigorously in [6]. This has been first tested with success on systems presenting a fluctuation driven first order transition, namely transitions which are expected to be continuous at a mean field level, but become first order when transitions are incorporated. Hence, Cardy has shown that the addition of weak disorder on a system of $N$ coupled Ising models ( a model presenting a first order transition driven by fluctuations) makes the system flow to $N$ decoupled Ising models [7]. This study has then been extended to the case of $N 3$-state Potts models by Pujol; the result was a non-Ising like second order transition (for $N>2$ ) [8]. The $q$-states Potts model with $q>4$ appears as a more natural model to test and analyse more deeply the effects of disorder, because the first order transition is now of mean field type, so much stronger. Nevertheless, there is no analytical approach able to control the effect of disorder on such systems. On the other hand, Chen et al. have investigated the 8-states potts Model using Monte Carlo simulations and confirmed the transition to be continuous but also found numerical values of the critical exponents consistent with those of the pure Ising model [9]. Yet, more recent numerical studies of Cardy and Jacobsen [10] and of Picco [11] are in a clear disagreement with the latter conclusion. They found a magnetic exponent $\beta / \nu$ which varies continuously with $q$. It has also been shown, using finite size scaling combined with conformal invariance, that the values of the central charges are related to one another by a factorization law $c\left(q=q_{1} \times q_{2}\right)=c\left(q_{1}\right)+c\left(q_{2}\right)$. Therefore, this measure is not able to distinguish between a non trivial random behavior for a $q=q_{1} \times q_{2}$ state random Potts model and two decoupled $q_{1}$ and $q_{2}$ state Potts models. This could suggest some links between a disordered $q=q_{1} \times q_{2}$-state Potts model and the corresponding disordered $q_{1}, q_{2}$-state potts models despite the magnetic exponents appear different for the 4, 8-state Potts models 10, 11.

Up to now, the only analytical results concern several $q$-state Potts models with disorder. We intend in this letter to analyse the case of two different Potts models with disorder. Therefore, we investigate analytically (in a perturbative sheme in powers of $(q-2)$ ) the behavior of two coupled $q_{1}$ and $q_{2}$-state potts models with $q_{1}, q_{2} \in\{2,3,4\}$ in order to compare it to the numerical results of the $q=q_{1} \times q_{2}$-state Potts model. We find a rather complex situation: When two different models are considered, there is a factorisation law and the models decouple. Therefore, our analysis confirms no apparent relations (at least perturbatively!) between the $q=q_{1} \times q_{2}$-state Potts model and the corresponding disordered $q_{1}, q_{2}$ state 
Potts models, despite their central charge to be similar. Nevertheless, when $q_{1}=q_{2}=3$ or 4 , the system flows in a strong coupling regime, despite the presence of disorder, what is in apparent contradiction with the Imry-Wortis argument.

Our model consists of one $q_{1}$-state Potts model coupled to a $q_{2}$-state Potts model by their energy operators $\left(q_{i} \in\{2,3,4\}\right)$. The Hamiltonian of the system has the following form

$$
H=H_{1}+H_{2}-g_{12} \int d^{2} x \varepsilon_{1} \varepsilon_{2}+m_{1} \int d^{2} x \varepsilon_{1}+m_{2} \int d^{2} x \varepsilon_{2} .
$$

$H_{1}, H_{2}$ are respectively the Hamiltonians of the pure $q_{1}$ and $q_{2}$ state Potts models, $m_{i}$ the reduced temperatures $(i=1,2), \varepsilon_{i}$ corresponds to the energy operators of the pure models and finally $g_{12}$ is the coupling constant associated to the interaction term. The partition function can hence be written as

$$
Z=\operatorname{Tr}_{s_{1, i}} \operatorname{Tr}_{s_{2, i}} e^{\left[S_{1}^{0}+S_{2}^{0}+g_{12} \int d^{2} x \varepsilon_{1} \varepsilon_{2}+m_{1} \int d^{2} x \varepsilon_{1}+m_{2} \int d^{2} x \varepsilon_{2}\right]}
$$

where $s_{1, i}$ is a spin operator of the $q_{1}$-state Potts model. Therefore, a correlation function $<O(0) O(R)>$, where $O$ is some local operator, is expanded perturbatively like:

$$
<O(0) O(R)>=<O(0) O(R)>_{0}+<S_{I} O(0) O(R)>_{0}+\frac{1}{2}<S_{I}^{2} O(0) O(R)>_{0}+\cdots
$$

where $<>_{0}$ means the expectation value taken with respect to $S_{1}^{0}+S_{2}^{0}$ and $S_{I}=\int d^{2} x \varepsilon_{1} \varepsilon_{2}$. The calculation of correlation functions can be performed with the Coulomb-gas representation [12. The central charge of a $q_{i}$-state Potts model is written as $c=\frac{1}{2}+\epsilon_{i}$, where $\epsilon_{i}$ can also be used as a short distance regulator for the integrals involved in correlation functions calculations. Then the limit $\epsilon \rightarrow 0$ corresponds to the Ising model while the Potts model is obtained for some finite value of $\epsilon$. The recent numerical results of Cardy et al. [10] have proved that the $(q-2)$ expansion gives accurate results for $q=3$ and good qualitative results for $q=4$ (with an error around $4 \%$ on the magnetic exponent of the disordered 4-state potts model).

When we have only one coupling constant $g_{0}$, its renormalisation is determined directly by a perturbative computation. $g$ is also given by the Operator Algebra (O.A.) producing

$$
g_{0} \int \varepsilon_{a}(z) \varepsilon_{b}(z) d^{2} z+\frac{1}{2}\left(g_{0} \int \varepsilon_{a}(z) \varepsilon_{b}(z) d^{2} z\right)^{2}+\cdots=g \int \varepsilon_{a}(z) \varepsilon_{b}(z) d^{2} z
$$

with $g=g_{0}+A_{2} g_{0}^{2}+\cdots$ where $A_{2}$ comes from the contraction

$$
\frac{1}{2} \int \varepsilon_{a}(z) \varepsilon_{b}(z) d^{2} z \int \varepsilon_{c}(z) \varepsilon_{d}(z) d^{2} z=A_{2} \int \varepsilon_{a}(z) \varepsilon_{b}(z) d^{2} z
$$

Therefore, in the limit $m_{i} \rightarrow 0$, the 2 -loop renormalisation group equation associated to (四) reads

$$
\beta\left(g_{12}\right) \equiv \frac{d g_{12}}{d \log r}=\frac{\epsilon_{1}+\epsilon_{2}}{2} g_{12}(r)+o\left(g_{12}^{3}\right)
$$


Here, $\epsilon_{i}=2-\Delta_{\varepsilon_{i}}$, with $\Delta_{\varepsilon_{i}}$ the physical dimension of the energy operator $\varepsilon_{i}$. For more details, we refer to references [12, 2]. In our notation, we have $\epsilon=0$ for the Ising model, $\epsilon=\frac{2}{5}$ for the 3 -state Potts model and $\epsilon=1$ for the 4 -state Potts model. From the equation (5), we clearly see that the system is driven in a strong coupling regime indicating probably a first order transition. A similar situation has already been encountered, when one couples two $q$-state Potts models [8]. Moreover, it can be proved exactly that we have a mass gap generation [13]. The equation (5) can be generalized for one Ising model coupled to several Potts models giving a new interesting fixed point structure [14].

We now add quenched randomness coupled to the local energy densities. This can be done by introducing in (1) position dependent random mass terms $m_{i} \rightarrow m_{i}(x)$ with $\overline{m_{i}(x)}=0$ and $\overline{m_{i}(x) m_{j}(y)}=\Delta_{i j} \delta(x-y) . \Delta_{i j}$ represents the $2 \times 2$ symmetric covariance matrix whose elements are strictly positive as it should be. If we have considered a diagonal covariance matrix corresponding to independent disorders for each models, then $\Delta_{12}$ would have been generated by the R.G. equations. We then apply the replicated method by introducing $n$ copies of the system and averaging Gaussian distributions for $m_{i}(x)$. We finally obtain the sum of $n q_{1}$ and $q_{2}$-state Potts models coupled by their energy densities. The Hamiltonian can be written as

$$
H=H_{1}+H_{2}-g_{12} \int d^{2} x \sum_{a} \varepsilon_{1}^{a} \varepsilon_{2}^{a}-\int d^{2} x \sum_{<i, j, a, b>} \Delta_{i j} \varepsilon_{i}^{a} \varepsilon_{j}^{b}
$$

where $i, j=1,2$ and $a, b$ runs from 1 to $n$ and $\langle\ldots>$ means that when $i=j$ then $a \neq b$. The hamiltonian (6) has four coupling constants. It is a generalization of the study of two Ising models with disorder [7] or two Potts models with disorder [8]. We now derive the 2-loops R.G. equations associated to (6). The procedure employed in a generalisation of (3). The methods we use are explained and detailed in ref. [2]. Nevertheless, since we have to compute correlations functions involving different energy operators, new integrals appear at one and two loops. Details of the algebra will be presented elsewhere [15]. Therefore, taking the replica limit $n \rightarrow 0$, the four beta functions are, at two loops

$$
\begin{aligned}
& \beta_{g_{12}} \equiv \frac{d g_{12}}{d \log l}=\frac{\epsilon_{1}+\epsilon_{2}}{2} g_{12}-g_{12}\left(\Delta_{11}+\Delta_{22}\right)+\frac{1}{2} g_{12}\left(\Delta_{1}^{2}+\Delta_{2}^{2}\right)+3 \Delta_{12}^{2} g_{12}+\Delta_{12} g_{12}^{2} \\
& \beta_{\Delta_{11}} \equiv \frac{d \Delta_{11}}{d \log l}=\epsilon_{1} \Delta_{11}-2 \Delta_{11}^{2}+2 g_{12} \Delta_{12}+2 \Delta_{11}^{3}-2 \Delta_{11} \Delta_{12} g_{12}-\Delta_{11} g_{12}^{2} \\
& \beta_{\Delta_{22}} \equiv \frac{d \Delta_{22}}{d \log l}=\epsilon_{2} \Delta_{22}-2 \Delta_{22}^{2}+2 g_{12} \Delta_{12}+2 \Delta_{22}^{3}-2 \Delta_{22} \Delta_{12} g_{12}-\Delta_{22} g_{12}^{2} \\
& \beta_{\Delta_{12}} \equiv \frac{d \Delta_{12}}{d \log l}=\frac{\epsilon_{1}+\epsilon_{2}}{2} \Delta_{12}+g_{12}\left(\Delta_{11}+\Delta_{22}\right)-\Delta_{12}\left(\Delta_{11}+\Delta_{22}\right) \\
& +\Delta_{12}\left[\frac{1}{2}\left(\Delta_{11}^{2}+\Delta_{22}^{2}\right)+\Delta_{12}^{2}-2 \Delta_{12} g_{12}-g_{12}^{2}\right]
\end{aligned}
$$


In these equations we have made the change $g_{12} \rightarrow 4 \pi g_{12}$ and $\Delta \rightarrow 4 \pi \Delta$. We have not considered replica symmetry breaking. Moreover, recent analytical and numerical results on disordered Potts models are in favor of a non replica symmetry breaking scenario [16]. When we couple two similar models (Ising or Potts), we have $\epsilon_{1}=\epsilon_{2}$ and $g_{12}=g, \Delta_{11}=\Delta_{12}=$ $\Delta_{22}=\Delta$ and the flow reduces to

$$
\begin{aligned}
& \frac{d g}{d \log l}=\epsilon g-2 g \Delta+g^{2} \Delta+4 g \Delta^{2} \\
& \frac{d \Delta}{d \log l}=\epsilon \Delta-2 \Delta^{2}+2 \Delta g+2 \Delta^{3}-g^{2} \Delta-2 \Delta^{2} g
\end{aligned}
$$

We recover in this case the results of Pujol [8].

We are looking for the fixed points by taking $\frac{d g_{12}}{d \log l}=\frac{d \Delta_{i j}}{d \log l}=0$. For the system (8), there are three fixed points

$$
g=0 ; \Delta=0
$$

the trivial one, and

$$
g=0 \quad ; \quad \Delta=\frac{\epsilon}{2}+\frac{\epsilon^{2}}{4}+O\left(\epsilon^{3}\right)
$$

the fixed point corresponding to a decoupling of the models and

$$
g=\frac{\epsilon^{2}}{4}+O\left(\epsilon^{3}\right) \quad ; \quad \Delta=\frac{\epsilon+\epsilon^{2}}{2}+O\left(\epsilon^{3}\right)
$$

a new non-trivial one mixing both models.

When we consider one Ising model coupled to a 3 or 4 -state Potts model, we take the limit $\epsilon_{1} \rightarrow 0$ and $\epsilon_{2}=\epsilon$, we find the following fixed points

$$
\begin{array}{r}
g_{12}^{*}=0 \quad ; \quad \Delta_{11}^{*}=\Delta_{22}^{*}=\Delta_{12}^{*}=0 \\
g_{12}^{*}=0 ; \Delta_{11}^{*}=0 ; \Delta_{12}^{*}=\frac{\epsilon}{2}+\frac{\epsilon^{2}}{4}+o\left(\epsilon^{2}\right) ; \Delta_{12}^{*}=0+o(\epsilon) \\
g_{12}^{*}=0 ; \Delta_{11}^{*}=0 ; \Delta_{12}^{*}=\frac{\epsilon}{2}+\frac{\epsilon^{2}}{4}+o\left(\epsilon^{2}\right) ; \Delta_{12}^{*}=\frac{\epsilon}{2}+o(\epsilon)
\end{array}
$$

We have also three fixed points, the trivial one (12), the one corresponding to a perfect decoupling of the disordered Ising and Potts models (13) and a new fixed point mixing a priori both models. Let us notice that $\Delta_{12}^{*}$ is undetermined at one loop and two loop calculations just enable to compute the first order in $\epsilon$. And finally, when one considers a 3-state coupled to a 4 - state Potts model, we only find the fixed points (12), (13).

In order to study the stability of each of these fixed points, we re-express the systems (匹) around the above solutions. Therefore, we write $g_{12}=g_{12}^{*}+\delta g_{12} ; \Delta_{i j}=\Delta_{i j}^{*}+\delta \Delta_{i j}$ and keep only the smallest order in $\epsilon$. We thus obtain a linear system $\delta \dot{X}=A X$ with $X=\left(\delta \Delta_{11}, \delta \Delta_{22}, \delta \Delta_{12}, \delta g_{12}\right)$. All the information concerning the stability is contained in 
the matrix $A$. For the R.G. equations (8), $A$ is only a $2 \times 2$ matrix. By calculating the eigenvalues of $A$ for each fixed points, we can show that the fixed points (9), (10) are unstable. For the fixed point (11), a numerical study of the flow (fourth order are needed for an analytical study of the stability of this fixed point!) shows that it is also unstable contrary to what was stated in [8]. Consequently, the system is driven in a strong coupling regime. Note that this result is surprising because it is in contradiction with Imry and Wortis arguments [5]. Nevertheless, we must not forget that we have done a perturbative analysis at two loops, so another non-perturbative fixed point can not be ruled out (or a perturbative one which needs three loops terms to be stabilized). Therefore, it would be very interesting to study the critical behavior of this model numerically.

A similar analysis can be repeated for $\epsilon_{1}=0$. Thus, we can see that (12) is unstable, that (13) is stable, and finally that (14) is stable only in the space $g_{12}=0$. In fact, the fixed point (14) appears as a generalisation of (10) for different minimal models. We have represented in Figure 1, the projection on the flow in the $\left(g_{12}, \Delta_{12}\right)$ plane for two different initial conditions (points $A_{0}$ and $B_{0}$ ). We clearly see that the flow first try to go away and then is driven by disorder at the origin corresponding to a perfect decoupling of the models. As it has been already noticed in [7], such a flow is unusual because it violates the $c$-theorem [17]. There are no corrections to the critical exponents which remain those of the corresponding decoupled disordered models. Note that in [8], a new stable fixed point was found (for $N>2$ 3-state Potts models), but with similar critical exponents that one disordered Potts model.

In this paper, we have analysed the behavior of two different coupled minimal models in presence of disorder. This constitutes a new example of first order transition driven by randomness in a second order one (see Figure 1). Moreover, we have shown than the models factorize, namely decouple. If we compare the critical exponent $\beta / \nu \sim 0.142$ found in [10] for the disordered 6 -state potts model, it appears clearly different from the one of the Ising or disordered 3-state Potts model. Therefore, there are no relations at a perturbative level between both models despite their central charges are similar. On the other hand, it would be very interesting to test numerically the behavior of two coupled $q_{1}, q_{2}$ Potts models with disorder and to analyse the non-perturbative area in relation with the $q_{1} \times q_{2}$-state Potts model. Indeed, for large $g_{12}$, we could imagine a cross-over phenomena in the system of two coupled minimal models. The case of two similar Potts models is special because it seems to violate the Imry-Wortis argument perturbatively. It deserves a more accurate analysis, either analytically from, for example, the integrable point of view or numerically. Finally, such an analysis can be extended to the case of $N$ Ising models to $M$ Potts models. We expect similar behaviors except, maybe, for the cases $N$ or $M=2$. 


\section{Acknowledgements}

I would like to thank Vl. S. Dotsenko for helpful suggestions and stimulating discussions. I also acknowledge M. Picco and P. Pujol for useful discussions.

\section{References}

[1] A. W. W. Ludwig, Nucl. Phys. B285, 97 (1987); Nucl. Phys. B330, 639 (1990).

[2] Vl. Dotsenko, M. Picco and P. Pujol, Phys. Lett B347, 113 (1995); Nucl. Phys. B 455, 701 (1995).

[3] E. Domany and S. Wiseman, Phys. Rev. E51, 3074 (1995); Phys. Rev. E52, 3469 (1995); M. Picco, Phys. Rev. B54, 14930 (1996).

[4] A. B. Harris, J. Phys. A7, 1671 (1974).

[5] Y. Imry and M. Wortis, Phys. Rev. B19, 3581 (1979); K. Hui and N. Berker, Phys. Rev. Lett. 62, 2507 (1989).

[6] M. Aizenman and J. Wehr, Phys. Rev. Lett. 62, 2503 (1989).

[7] J. L. Cardy, J. Phys. A26, 1897 (1996).

[8] P. Pujol, Euro. Phys. Lett. 35, 283 (1996).

[9] S. Chen, A.M. Ferrenberg and D. P. Landau, Phys. Rev. Lett. 69, 1213 (1992); Phys. Rev. E52, 1377 (1995).

[10] J. L. Cardy and J. L. Jacobsen, cond-mat/9705038.

[11] M. Picco, cond-mat/9704221] and private communication.

[12] Vl. S. Dotsenko and V.A. Fateev, Nucl. Phys. B240, 312 (1984), B251, 691 (1985).

[13] L. Vaysburd, Nucl. Phys. B446, 387 (1995).

[14] P. Simon, cond-mat/9705292, to be published in Phys. Lett. B.

[15] P. Simon, in preparation

[16] Vl. S. Dotsenko et al., in preparation.

[17] A. B. Zamolodchikov, Sov. J. Nucl. Phys. 46, 6 (1987); Advances Studies in Pure Mathematics 19, 641 (1989). 


\section{FIGURE CAPTION}

FIG.1 : The projection of two flows in the $\left(g_{12}, \Delta_{12}\right)$ plane for one Ising model coupled to a 3-state Potts models with disorder. $A_{0}, B_{0}$ correspond to two different initial conditions. We clearly see that the flow is driven by randomness at the origin. It corresponds to a decoupling regime.

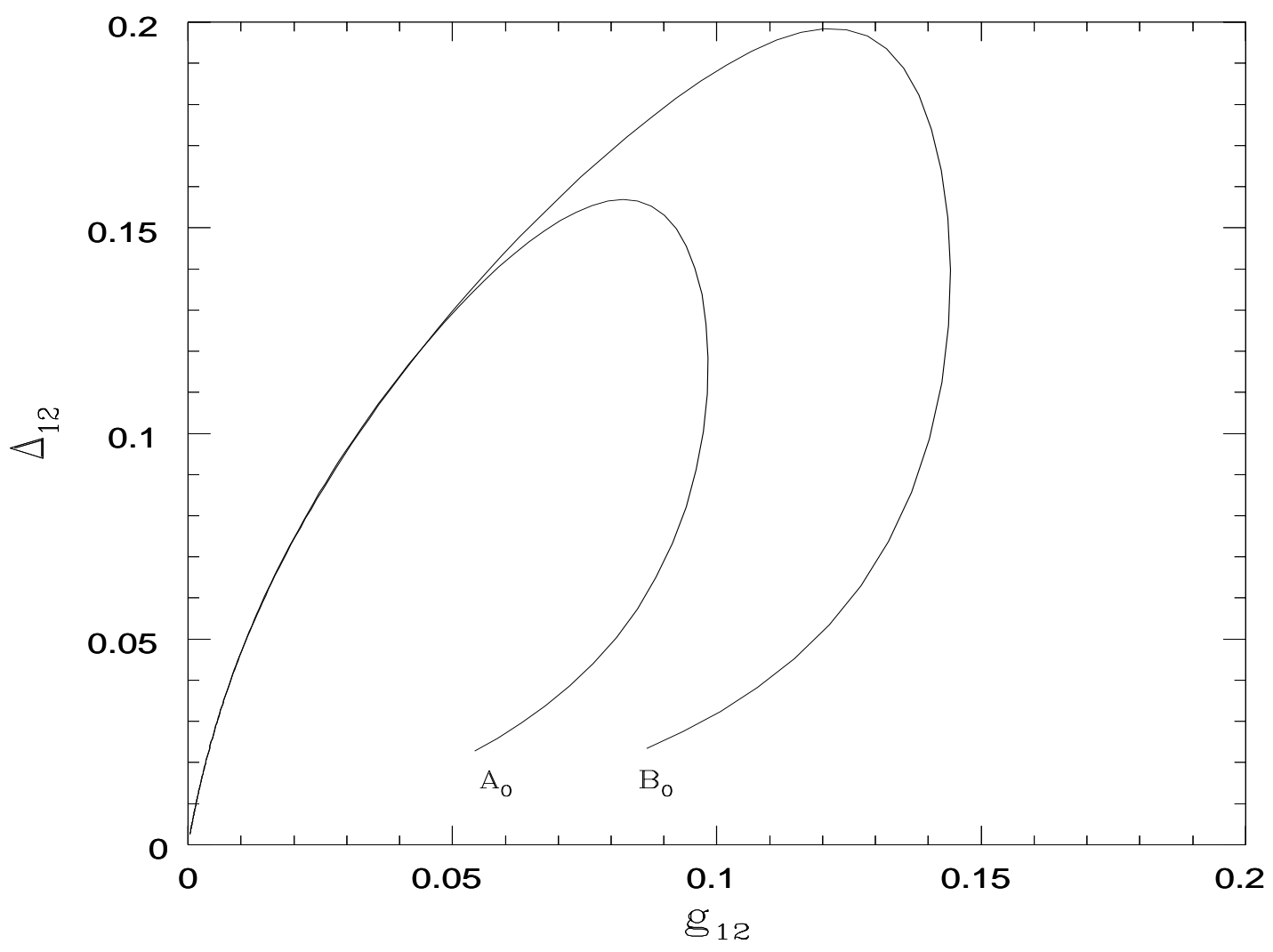

